\title{
OSA ist häufig, aber wenig symptomatisch
}

Fragestellung: Wie häufig ist obstruktive Schlafapnoe (OSA) in Island und wie ist die Symptomatik?

Hintergrund: Die OSA wurde noch vor 30 Jahren als seltene Erkrankung angesehen und war vielen Ärzten unbekannt. Heute werden ganze Kongresse darüber veranstaltet und sie dient als Erklärung für viele klinische Symptome. Zweifellos hat der Anstieg der Adipositas zu einer Zunahme der OSA geführt, doch könnten auch Änderungen der diagnostischen Methoden und der Definitionskriterien dazu beigetragen haben.

Patienten und Methoden: Bei den Patienten handelt es sich um eine repräsentativ ausgewählte Kohorte der isländischen Bevölkerung, die bereits zweimal $(1990,2000)$ im Rahmen des Respiratory Health Survey der Europäischen Union (ECRHS) untersucht wurde. Von 522 nun zum 3. Mal aufgeforderten Personen verweigerten 40 die Teilnahme, waren 8 weggezogen, 2 gestorben, 6 nicht mehr auffindbar und fanden 2 keine Zeit, sich ein nochmal untersuchen zu lassen. Stabile isländische Verhältnisse eben. Die Teilnehmer füllten Fragebögen zu Symptomatik und Schlafverhalten aus und unterzogen sich einer Polysomnografie

Originalie

Arnardottir ES, Bjornsdottir E, Olafsdottir KA et al. Obstructive sleep apnoea in the general population: highly prevalent but minimal symptoms. Eur Respir J. 2016 Jan;47(1):194-202. und einem psychomotorischen Vigilanztest. 415 Personen von 40-Jahren lieferten auswertbare Ergebnisse.

Ergebnisse: Von den 415 Probanden hatten 15 bereits früher die Diagnose eines OSA bekommen und standen unter entsprechender Behandlung. Das mediane Alter lag bei 54,7 $\pm 6,8$ Jahren, der BMI bei 28,2 $\pm 5,0 \mathrm{~kg} /$ $\mathrm{m}^{2} .16,6 \%$ waren aktive Raucher, $30,6 \%$ bekannte Hypertoniker. Jeweils 3\% hatten Typ-2-Diabetes, Zustand nach Schlaganfall und KHK. Anhand des Apnoe-Hypopnoe-Index (AHI) hatten 100 Personen (25\%) leichte, 52 (13\%) mäßige und 12 (3\%) schwere OSA, bei 236 (59\%) wurde keine OSA festgestellt.

In einer linearen Regressionsanalyse waren Geschlecht, Alter und BMI signifikante Variable für die Höhe des AHI und erklärten 23,5\% der AHI-Varianz. Der Schweregrad des OSA korrelierte aber nicht mit der Raucheranamnese oder einer Hypertonie. Personen mit mäßiger oder schwerer OSA litten nicht wahrscheinlicher unter metabolischen und kardiovaskulären Erkrankungen als solche ohne oder mit leichter OSA. Besonders überraschend war es, dass die Symptome des Schlafmangels wie Tagesmüdigkeit oder Konzentrationsstörungen nur mit dem Symptom des gewohnheitsmäßigen Schnarchens, nicht aber mit den Kategorien des AHI korrelierten. Bei den objektiven Tests von Vigilanz, Reaktionszeit und Fehlerhäufigkeit gab es zwar ein signifikante Unterschiede zwischen Personen mit und ohne OSA, diese wurden aber nur durch die kleine Gruppe mit $A H I \geq 30(n=12)$ verursacht. Personen mit niedriger und mäßiger OSA schnitten in den objektiven Vigilanztests nicht signifikant schlechter ab als die ohne.

Schlussfolgerung: Nach den aktuellen Definitionskriterien ist die OSA eine häufige Störung, die aber offensichtlich kaum Konsequenzen für das Befinden der Betroffenen hat.

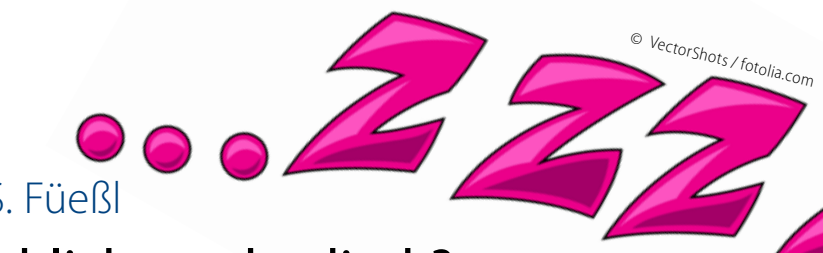

- Kommentar von Professor Dr. med. Hermann S. Füeßl

\section{Prävalenz-Anstieg der OSA hauptsächlich methodisch?}

Nach den Ergebnissen der Untersuchung liegt die Prävalenz der OSA bei Personen im mittleren Lebensalter zwar fast bei $20 \%$, doch besitzt dieser Befund für die überwiegende Mehrzahl keine gesundheitliche Relevanz. Die Relevanz für den Schlafpartner mag erheblich sein, für die Entwicklung einschlägiger Symptome wie Tagesmüdigkeit, Konzentrationsstörungen, Hypertonie oder koronare Herzkrankheit spielt die OSA bis auf die kleine Gruppe mit sehr großem AHI kaum eine Rolle. Dabei handelt es sich nicht nur um ein subjektives Gefühl, sondern waren Defizite der Vigilanz bei Personen mit leichtem bis mäßigem OSA nicht einmal in Testsituationen nachweisbar. Die bloße Feststellung einer OSA in der Polysomnografie sollte also nicht automatisch zu einer (oft wenig akzeptierten) therapeutischen Maßnahme führen, da sich das Befinden der Patienten kaum bessern dürfte. Insofern besitzt die Untersuchung hohe praktische Relevanz, da sie der in den letzten Jahren beobachteten Tendenz widerspricht, viele Befindlichkeitsstörungen auf das Konto einer OSA zu schreiben.
Die fast „inflationäre“ Zunahme der OSA in den letzten Jahren spiegelt nach Ansicht der Autoren weniger eine echte Zunahme der nächtlichen Atemstörung infolge der Adipositas wider, sondern wird ganz wesentlich durch geänderte Messmethoden und Scores getriggert. Es liegt in der Natur der Sache, dass jedes Fachgebiet der Medizin versucht, sich durch eine möglichst große Häufigkeit der behandelten Erkrankungen in den Vordergrund zu schieben. Insofern leistet die Studie einen wertvollen Beitrag, um hier Manches wieder auf dem Boden der Tatsachen zurückzuholen. Dennoch soll nicht verschwiegen werden, dass es sich um eine Querschnittsanalyse handelt, die keine Aussagen über die gesundheitlichen Effekte einer langfristig bestehenden OSA machen kann.

Prof. Dr. med. Hermann S. FüeßI, München 